\title{
Isolation of circulating tumor cells and detection of EGFR mutations in patients with non-small-cell lung cancer
}

\author{
QI ZHANG ${ }^{1}$, JINGYING NONG $^{1}$, JINGHUI WANG $^{1}$, ZHUOHONG YAN $^{2}$, LING YI $^{2}$, \\ $\mathrm{XIN} \mathrm{GAO}^{2}$, ZHIDONG LIU $^{3}$, HONGTAO ZHANG $^{2}$ and SHUCAI ZHANG ${ }^{1}$ \\ Departments of ${ }^{1}$ Oncology, ${ }^{2}$ Central Laboratory and ${ }^{3}$ Thoracic Surgery, Beijing Chest Hospital, \\ Capital Medical University, Beijing Tuberculosis and Thoracic Tumor Research Institute, Beijing 101149, P.R. China
}

Received March 15, 2018; Accepted November 2, 2018

DOI: $10.3892 / \mathrm{ol} .2019 .10016$

\begin{abstract}
The aim of the present study was to develop a procedure for the isolation of circulating tumor cells (CTCs), and to evaluate its application in the detection of epidermal growth factor receptor (EGFR) mutations, and potential heterogeneity in patients with non-small-cell lung cancer (NSCLC). Peripheral blood samples were collected from 91 patients with lung cancer, 10 patients with benign disease and 10 healthy volunteers. CTCs were enriched by positive immunomagnetic separation, detected by immunocytochemistry, and processed for single-cell capture. Pure CTC DNA was amplified, and the EGFR gene was analyzed using the amplification refractory mutation system (ARMS) and digital polymerase chain reaction (dPCR). The CTC capture rate in patients with lung cancer was $61.5 \%(56 / 91)$, whereas no CTCs were detected in patients with benign lung disease or in healthy volunteers. The CTC-positive detection rates were $69.3 \%(52 / 75)$ and $25.0 \%(4 / 16)$ in patients with TNM stage III and IV disease, respectively. Markedly more CTCs were captured from patients with small-cell lung
\end{abstract}

Correspondence to: Dr Hongtao Zhang, Department of Central Laboratory, Beijing Chest Hospital, Capital Medical University, Beijing Tuberculosis and Thoracic Tumor Research Institute, 97 Machang Road, Tongzhou, Beijing 101149, P.R. China

E-mail: zhtbeijing@163.com

Dr Shucai Zhang, Department of Oncology, Beijing Chest Hospital, Capital Medical University, Beijing Tuberculosis and Thoracic Tumor Research Institute, 97 Machang Road, Tongzhou, Beijing 101149, P.R. China

E-mail: sczhang6304@163.com

Abbreviations: CTCs, circulating tumor cells; EGFR, epidermal growth factor receptor; EpCAM, epithelial cell adhesion molecule; $\mathrm{CK}$, cytokeratin; FITC, fluorescein isothiocyanate; CNV, copy number variation; ARMS, amplification refractory mutation system; WGA, whole-genome amplification; COSMIC, The Catalogue of Somatic Mutations in Cancer; NSCLC, non-small-cell lung cancer

Key words: circulating tumor cells, epidermal growth factor receptor mutation, homogeneity, non-small-cell lung cancer cancer compared with patients with other types of cancer. In patients who were positive for EGFR mutations, the detection rate of these mutations was low $(16.67 \%, 2 / 12)$, at the single CTC level. The sensitivity increased as the number of CTCs per sample increased. A total of four patients displayed consistent detection of EGFR mutations at the 10-cell level, and one patient exhibited a clear, inconsistent and rare mutation (G719x) between CTCs. A simplified technique for isolating CTCs from blood was established, though multiple CTCs were required to sensitively detect mutations in these cells. The detection of EGFR mutations in CTCs and tissue specimens was generally homogeneous, and therefore, the CTC-level mutation analysis may potentially contribute to the discovery of heterogeneous mutations.

\section{Introduction}

Lung cancer is one of the most common malignancies, and the leading cause of cancer-associated mortality worldwide. The mortality of lung cancer has decreased over the past decade, and the current 5 -year survival rate is $\sim 18 \%$. These alterations are attributed to a decrease in tobacco use, the progress in lung cancer screening with low-dose computed tomography, and improvements in treatment in recent years (1). Novel targeted treatments for advanced non-small-cell lung cancer (NSCLC) based on driver mutations and immune checkpoints are continuously being developed (2-4). Epidermal growth factor receptor (EGFR) mutations are the most frequent driver mutations detected in lung adenocarcinomas and exhibit clinically therapeutic implications. The occurrence of these mutations varies between races, ranging from $\sim 14 \%$ in Caucasian patients to almost $50 \%$ in Asian patients, particularly those with adenocarcinoma $(5,6)$. Currently, the detection of EGFR mutations relies mainly on tissue specimens, and the identification of a mutation-positive tissue is the premise of targeted therapy. Tests examining circulating tumor DNA (ctDNA) are also used in the clinic. An early report detected EGFR mutations in circulating tumor cells (CTCs) using a microfluidic device, and achieved high rates of detecting EGFR-activating mutation (7).

In the present study, a fast, simple CTC isolation technique was established based on the CellSearch ${ }^{\circledR}$ principle $(8,9)$, which may simplify the verification of the EGFR status in CTCs. The 
status of EGFR mutations was evaluated by isolating blood epithelial cells from patients with advanced NSCLC prior to treatment with tyrosine kinase inhibitors (TKIs). The isolation and analysis of a few mixed CTCs significantly improved the detection rate of EGFR mutations compared with detection using single CTCs, and isolated CTCs were potentially useful for analyzing the heterogeneity of mutations.

\section{Materials and methods}

Patients and ethics. The study was approved by the Beijing Chest Hospital Committee on the Use of Humans as Experimental Subjects (Beijing China). In addition, all participants provided written informed consent. Patients were diagnosed with biopsy-validated lung cancer in clinical phase III or IV. The 57 male and 34 female patients had an age range of 32 to 87 years and a mean age of 62.3 years. Blood samples $(4.5 \mathrm{ml})$ were collected into Vacuette EDTA anticoagulant tubes (Greiner Bio-One GmbH, Kremsmünster, Austria) from 91 untreated patients with lung cancer at the Beijing Chest Hospital, and processed within $2 \mathrm{~h}$ using an autoMACS Pro instrument (Miltenyi Biotec GmbH, Bergisch Gladbach, Germany).

CTC enrichment and single-cell retrieval. Erythrocyte lysates were prepared from blood samples using BD FACS Lysing Solution (BD Biosciences, San Jose, CA, USA). Following red blood cell depletion, the Fc receptor reagent (cat. no. 120-000-442; Miltenyi Biotec GmbH) and cluster of differentiation CD326 microbeads (cat. no. 130061101; Miltenyi Biotec $\mathrm{GmbH}$ ) were used according to the manufacturer's protocol. The cell suspension was subsequently loaded onto an autoMACS Pro instrument, and CD326+ and CD326- fractions were obtained. The CD326+ cell fraction was stained with anti-epithelial cell adhesion molecule (EpCAM)-phycoerythrin (cat. no. 565399; BD Biosciences), three types of anti-cytokeratin-fluorescein isothiocyanate antibody (cat. no. ab870010, Abcam, Cambridge, UK; cat. no. ab72813, Abcam; cat. no. 53-9898, eBioscience; Thermo Fisher Scientific, Inc., Waltham, MA, USA), anti-CD45allophycocyanin antibodies (cat. no. ab28106; Abcam), and Hoechst 33342 (cat. no. C0030; Beijing Solarbio Science \& Technology Co.,Ltd., Beijing, China) to distinguish cancerous cells from healthy leukocytes. Individual CTCs (EpCAM+, Hoechst+, anti-cytokeratin+, anti-CD45-) were isolated from leukocytes (anti-CD45+, Hoechst+, anti-cytokeratin-, EpCAM-) using a fluorescence microscope (Nikon Corporation, Tokyo, Japan) with a robotic micromanipulator (CellEctor; Molecular Machines \& Industries GmbH, Eching, Germany). A total of three commercially available lung cancer adenocarcinoma cell lines (NCI-H2009, NCI-H1975, and HCC-827) were purchased from The American Type Culture Collection (Manassas, VA, USA) for spiking experiments and mutation analyses. For spiking experiments, CTCs were picked up by applying CellEctor and spiked into $4.5 \mathrm{ml}$ blood.

DNA amplification. The selected CTCs were used for whole-genome amplification (WGA) and quantification using a REPLI-g ${ }^{\circledR}$ Single Cell kit (cat. no. 150343; Qiagen
GmbH, Hilden, Germany). The procedure was performed according to the manufacturer's protocol. DNA was quantified using a NanoDrop 2000c spectrophotometer (Thermo Fisher Scientific, Inc., Wilmington, DE, USA), according to the manufacturer's protocol. The amplified DNA was verified by polymerase chain reaction (PCR) using the Multiplex PCR kit (cat no. 206143; Qiagen $\mathrm{GmbH}$ ) and eight pairs of primers specific for housekeeping genes were designed. The primers used were follows: CYB5A forward, 5'-GGCAAC GCTTAGACTCTGTGTG-3' and reverse, 5'-CTGCCCTTG GCCTAACTAACCT-3'; PRPH forward, 5'-GTTCCTCAA GAAGCTGCACGAG-3' and reverse, 5'-CGTTAGACTCTG GATCTGGCGT-3'; GABARAPL2 forward, 5'-CCAGCC AATTCATGAGTCGGTG-3' and reverse, 5'-CCTGACAAC TCGCAAGTAGCAC-3'; ACTG1 forward, 5'-GCTCAA TGGGGTACTTCAGGGT-3' and reverse, 5'-GTGGACGTT ACGTAAAAGGCCC-3'; NDUFA7 forward, 5'-TGCTCT GGATGTGAAGATGCCA-3' and reverse, 5'-TTCCAGGTA AATCCAGCCCAGG-3'; UQCRC1 forward, 5'-CAGCCA GTCAGCATCATCCAAC-3' and reverse, 5'-GAAAGC CGGATTGCGGTAACAT-3'; MYC forward, 5'-GGATAG CTCTGCAAGGGGAGAG-3' and reverse, 5'-TCGTCG CAGTAGAAATACGGCT-3'; and MIF forward, 5'-AGA AGTCAGGCACGTAGCTCAG-3' and reverse, 5'-GGC ACGTTGGTGTTTACGATGA-3'.

Sequence and copy number variation (CNV) analyses. For the analysis of CNVs, DNA was quantitatively amplified from $2.5 \mathrm{ng} / \mathrm{ml}$ WGA products for library preparation and barcoded with a Nextera Index kit (Illumina, Inc., San Diego, CA, USA). The low-quality reads were removed from the obtained data using the Illumina procedure following data quality control, and Burrows-Wheeler Alignment Tool (version 0.7.15) $(10,11)$ was used to compare the sequences to the human genome (hg19). The data normalized by unique reads were considered CNVs. Our test samples used white blood cells from the same patient as a reference for comparison.

EGFR mutation detection. EGFR mutations were detected using a quantitative fluorescence PCR instrument (Cobas z480; Roche Diagnostics, Basel, Switzerland) and an EGFR detection kit (human EGFR gene mutations fluorescence PCR diagnostic kit; Amoy Diagnostics Co., Ltd., Xiamen, China) according to the manufacturers' protocols. EGFR mutations were also analyzed by digital PCR (dPCR; ABI-QuantStudio 3D, Applied Biosystems; Thermo Fisher Scientific, Inc.), according to the protocols for the Dual Flat Block GeneAmp PCR System 9700 (Thermo Fisher Scientific, Inc.); dPCR primers and probes (cat no. A44177; Assay ID, HS000000026_rm; Thermo Fisher Scientific, Inc.) for EGFR mutation detection were purchased from Applied Biosystems. QuantStudio $^{\mathrm{TM}}$ 3D AnalysisSuite ${ }^{\mathrm{TM}}$ software version 3.1.2 (Thermo Fisher Scientific, Inc.) was used for data acquisition and analysis.

Statistical analyses. Counts were compared using the $\chi^{2}$ test. Continuous data were compared using Student's t-test if the distribution of samples was normal, or nonparametric tests (the Mann-Whitney U test) for comparisons between two groups. For each test, two-sided $\mathrm{P}<0.05$ was considered to indicate a 
$\mathbf{A}$

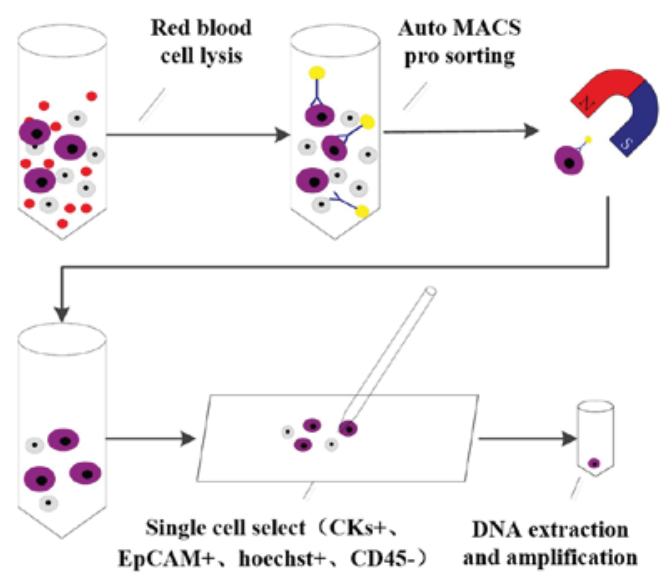

C

\begin{tabular}{llllll}
\hline & \multicolumn{5}{c}{ Spiked cell number } \\
& 2 & 5 & 10 & 20 & 40 \\
\hline Exp1 & 1 & 3 & 6 & 15 & 33 \\
Exp2 & 2 & 3 & 7 & 14 & 34 \\
Exp3 & 1 & 4 & 6 & 14 & 32 \\
Exp4 & 1 & 3 & 8 & 15 & 35 \\
\hline
\end{tabular}

B

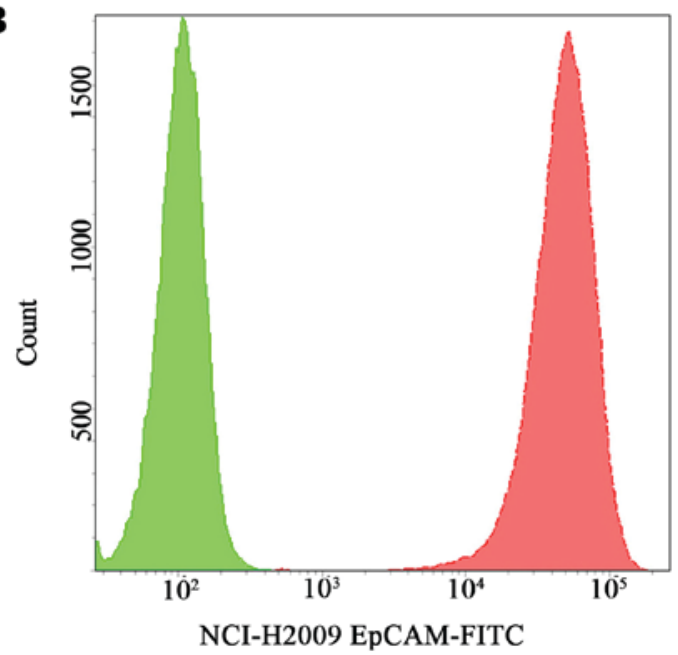

D

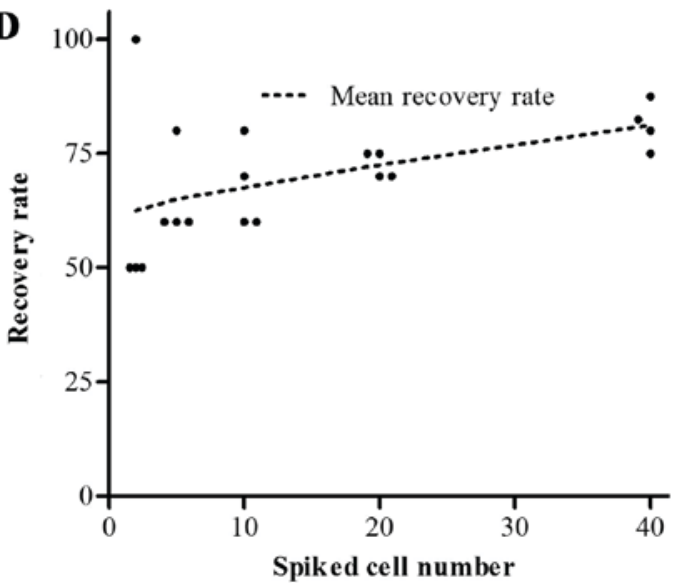

Figure 1. Isolation of spiked cancer cells from peripheral blood based on epithelial markers. (A) Peripheral blood was enriched by depleting red and white blood cells using the autoMACS Pro instrument. The remaining cells were stained with a panel of fluorescent antibodies. CTCs were defined as $\mathrm{CK}+/ \mathrm{EpCAM}+/ \mathrm{DAPI}+/ \mathrm{CD} 45$ - cells, and micromanipulation was performed under a fluorescence microscope. (B) Expression of EpCAM on the surface of spiked H2009 lung cancer cells. (C) Tabulated and (D) graphical representation of the recovery rates for four independent spiking experiments EpCAM, epithelial cell adhesion molecule; CK, cytokeratin; CTC, circulating tumor cell; Exp, experiment; FITC, fluorescein isothiocyanate; CD; cluster of differentiation.

statistically significant difference. All statistical manipulations were performed using the SPSS version 18 for Windows software system (SPSS, Inc., Chicago, IL, USA).

\section{Results}

A simplified technique to yield high-purity epithelial tumor cells from blood. An H2009 lung cancer cell line that stably expressed both EpCAM and cytokeratins (CKs) was selected for spiking experiments to establish an approach for isolating tumor cells from blood. The aim was to optimize the collection of pure CTCs for further molecular characterization at the individual cell level. An illustration of the CTC capture method is displayed in Fig. 1A and includes the following steps: i) Ammonium chloride-based red blood cell lysis; ii) enrichment of disseminated cancer cells using magnetically coated anti-EpCAM beads; and iii) micromanipulation to obtain single cells. In the present study, two epithelium-specific staining procedures (using EpCAM and CK antibodies) were performed to identify cancer cells, including EpCAM bead enrichment followed by staining with a monoclonal antibody against EpCAM that bound well to the cancer cell surface (Fig. 1B). The recovery rates of H2009 cells ( $\geq 1$ cell) ranged from $62.5-83.8 \%$ in four independent experiments (Fig. 1C and D) and increased when increasing the number of spiked cancer cells via the precise recovery of cells using micromanipulation. Similar capture efficiencies were achieved using cell lines exhibiting relatively low EpCAM expression levels, including the lung cancer cell lines H1975 and HCC-827. Using EpCAM and CK double staining, epithelial marker-positive cells were not detected in samples from 20 healthy subjects, from 10 patients with tuberculosis and from 10 patients with other nonmalignant lung diseases. This protocol was subsequently used to obtain rare CTCs from clinical samples.

Efficient real-time isolation of CTCs from patients with advanced lung cancer. A total of 91 patients with advanced lung cancer were enrolled for the CTC analysis as described in this study, between March 2016 and October 2017. Patient characteristics were recorded, and ages ranged from 32-83 years; $63 \%$ were male and $66 \%$ had adenocarcinoma. Patients with TNM stage III and IV diseases represented 18 and $82 \%$ of the lung cancer cohort, respectively. 
Table I. Correlation between captured CTC counts and clinical characteristics among patients with lung cancer prior to treatment.

\begin{tabular}{|c|c|c|c|c|c|c|c|}
\hline \multirow[b]{2}{*}{ Factor } & \multirow{2}{*}{$\begin{array}{c}\text { Patients with } \\
\text { CTC }(\text { Cutoff } \geq 1)(\%)\end{array}$} & \multicolumn{6}{|c|}{ CTC count $(\%)$} \\
\hline & & 0 & 1 & $2-3$ & $4-5$ & $6-10$ & $>10$ \\
\hline All patients $(\mathrm{n}=91)$ & $56(61.5)$ & $35(38.5)$ & $10(10.99)$ & $15(16.5)$ & $11(12.1)$ & $6(6.6)$ & $14(15.4)$ \\
\hline \multicolumn{8}{|l|}{ C-stage } \\
\hline III (n=16) & $4(25.0)$ & $12(75)$ & $0(0)$ & $1(6.3)$ & $2(12.4)$ & $0(0)$ & $1(6.3)$ \\
\hline IV $(n=75)$ & $52(69.3)$ & $23(30.7)$ & $10(13.3)$ & $14(18.7)$ & $9(12)$ & $6(8)$ & $13(17.3)$ \\
\hline \multicolumn{8}{|l|}{ Histological cell type } \\
\hline Adeno (n=60) & $36(60.0)$ & $24(30)$ & $7(11.7)$ & $12(20)$ & $5(8.3)$ & $5(8.3)$ & $7(11.7)$ \\
\hline $\mathrm{SCC}(\mathrm{n}=16)$ & $10(62.5)$ & $6(37.5)$ & $2(12.5)$ & $2(12.5)$ & $2(12.5)$ & $1(6.3)$ & $3(18.7)$ \\
\hline $\operatorname{SCLC}(n=9)$ & 7 (77.8) & $2(22.2)$ & $0(0)$ & $0(0)$ & $4(44.4)$ & $0(0)$ & $3(33.3)$ \\
\hline Other $(n=6)$ & $3(50.0)$ & $3(50)$ & $1(33.3)$ & $1(33.3)$ & $0(0)$ & $0(0)$ & $1(33.3)$ \\
\hline \multicolumn{8}{|l|}{ Smoking history } \\
\hline Yes $(\mathrm{n}=61)$ & $36(59.0)$ & $25(41)$ & $3(4.9)$ & $11(18.0)$ & $8(13.1)$ & $5(8.2)$ & $9(14.8)$ \\
\hline No $(n=30)$ & $20(66.7)$ & $10(33.3)$ & $7(23.3)$ & $4(13.3)$ & $3(10)$ & $1(3.3)$ & $5(16.7)$ \\
\hline \multicolumn{8}{|l|}{ Tumor size } \\
\hline$<3 \mathrm{~cm}(\mathrm{n}=39)$ & 19 (48.7) & $20(51.3)$ & $4(10.3)$ & $5(12.8)$ & $2(2.2)$ & $3(5.1)$ & $5(12.8)$ \\
\hline$>3 \mathrm{~cm}(\mathrm{n}=52)$ & 37 (71.1) & $15(28.9)$ & $6(11.5)$ & $10(19.2)$ & $9(17.3)$ & $3(5.8)$ & $9(17.3)$ \\
\hline
\end{tabular}

CTC, circulating tumor cell; Adeno, adenocarcinoma; SCC, squamous cell carcinoma; and SCLC, small-cell lung cancer.

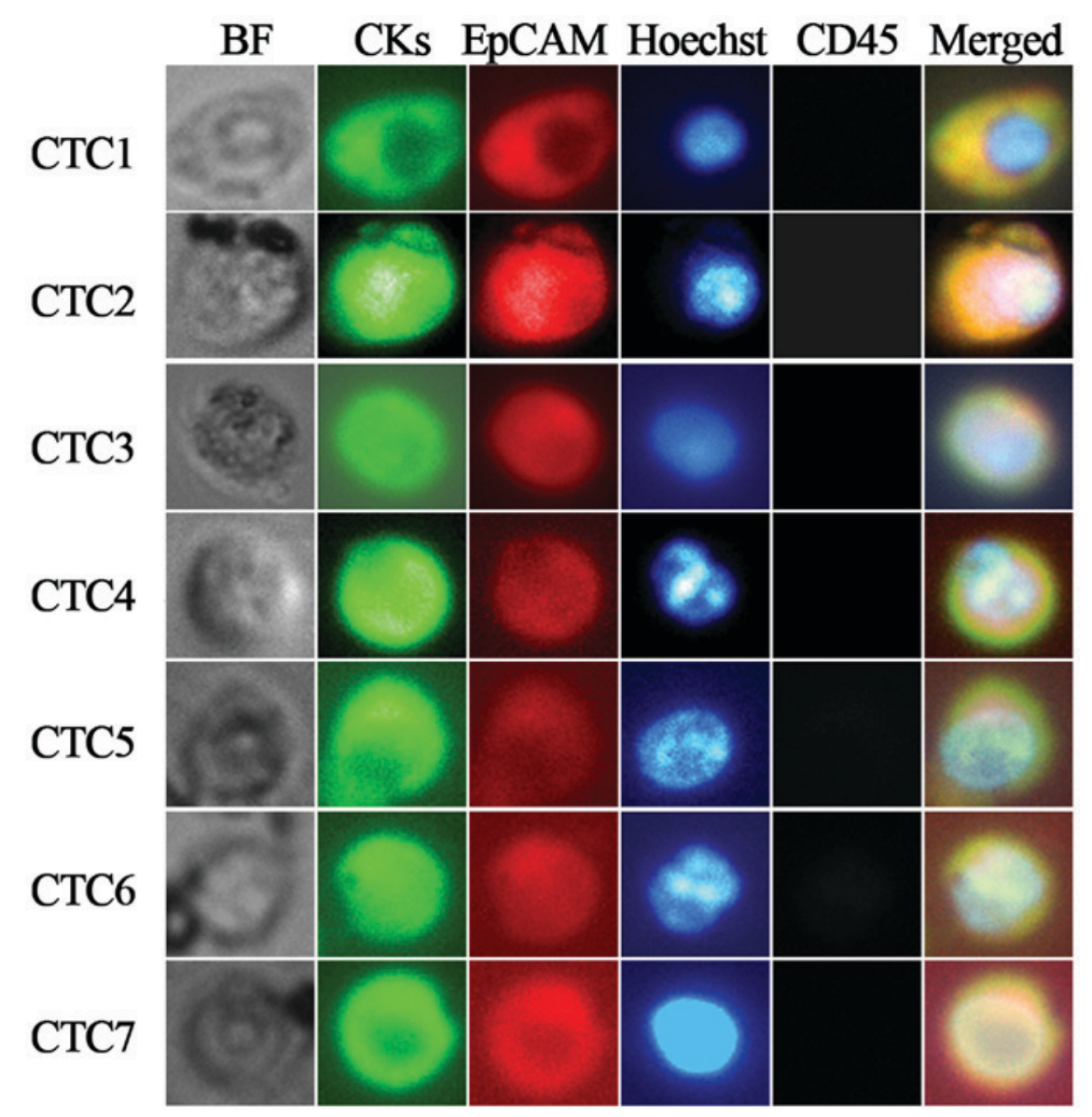

Figure 2. Representative images of individual CTCs isolated from $4.5 \mathrm{ml}$ of blood from patients with advanced lung cancer (magnification, $\mathrm{x} 400$ ). CTCs 1-4 were captured from four different patients with lung cancer; CTCs 5-7 were captured from the same patient with lung cancer. Tumor cells were identified as EpCAM-positive (red-PE), pan-CK-positive (green-FITC), CD45-negative (gray-APC) and Hoechst 33342-positive (blue) cells. CTC, circulating tumor cell; EpCAM, epithelial cell adhesion molecule; CK, cytokeratin; PE, phycoerythrin; FITC, fluorescein isothiocyanate; APC, allophycocyanin; BF, bright field; $\mathrm{CD}$, cluster of differentiation. 

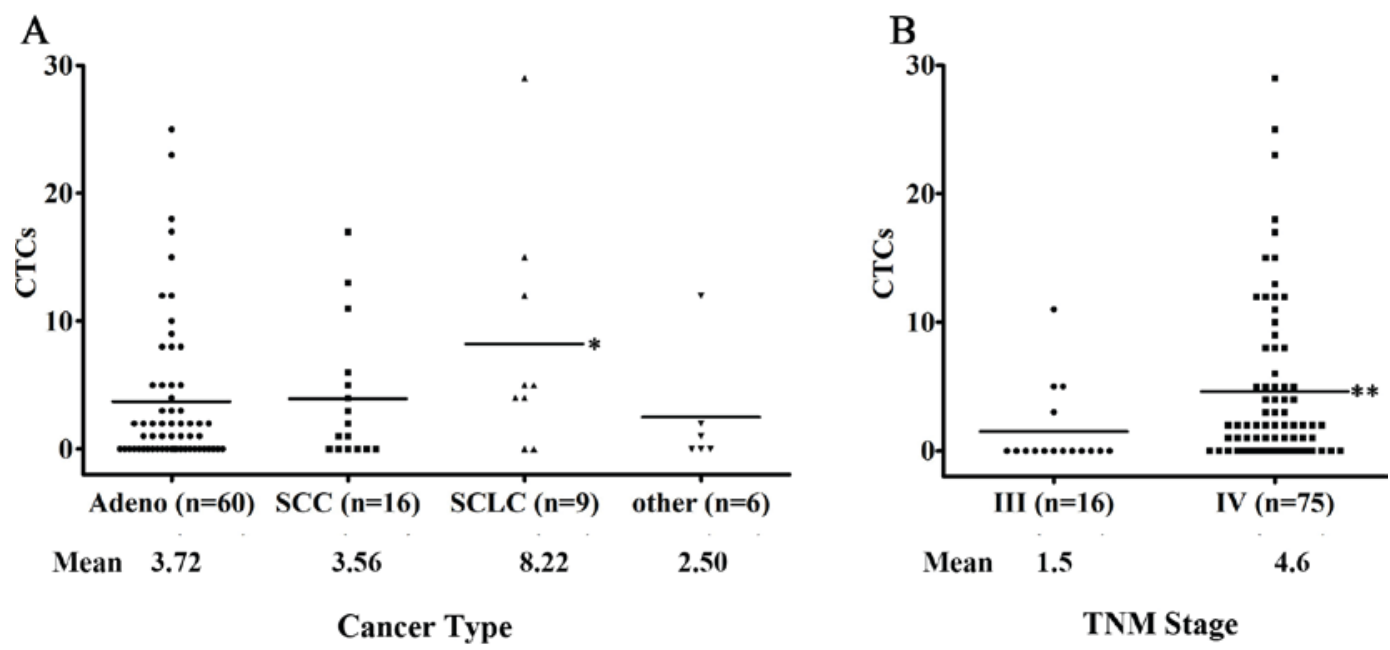

Figure 3. Association of CTC counts among all patients based on tumor histology and staging. The mean number of detected CTCs is illustrated. (A) Patients with SCLC had the highest CTC counts and a CTC detection rate of $77.8 \%$ (7 of 9 patients; median 5; mean 8.22) compared with patients with NSCLC or other types of metastatic disease. (B) TNM Stage IV NSCLC was significantly associated with higher CTC numbers (Mann-Whitney test, $\mathrm{P}=0.0081$ ). At least 1 CTC was detected in $52(69.3 \%)$ of the 75 patients with TNM stage IV disease. At least 1 CTC was detected in four (25.0\%) of the 16 patients with TNM stage III disease. CTC, circulating tumor cell; SCLC, small-cell lung cancer; NSCLC, non-small-cell lung cancer; SCC, squamous cell carcinoma; Adeno, adenocarcinoma; TNM, tumor, node, metastasis.

Among the total population, 56 patients (61.5\%) exhibited positive CTC counts at baseline, with the detection of at least one CTC in $4.5 \mathrm{ml}$ of peripheral blood (range, 1 to 29; median 2; mean 4.60). All identified cells were single CTCs, and no clusters were detected. Representative images of CTCs from individual patients are illustrated in Fig. 2. The prevalence of CTCs prior to treatment and the association with patient characteristics are listed in Table I. i) Stage IV NSCLC was significantly associated with higher CTC numbers (Mann-Whitney test, $\mathrm{P}=0.0081$ ). At least one CTC was detected in $52(69.3 \%)$ of the 75 patients with stage IV disease. In addition, at least one CTC was detected in four (25.0\%) of the 16 patients with stage III NSCLC (Fig. 3), and CTCs were also detected in one of the four patients with stage IIIa NSCLC, in whom two CTCs were captured. Detectable CTCs, albeit at lower numbers, positively correlated with bone and brain metastases compared with metastatic disease at other sites. Among 56 patients with CTCs, patients with bone, brain, liver and kidney metastasis accounted for $31 / 56$ (55.3\%), 25/56 (44.6\%), 15/56 (26.8\%) and 9/56 (16.1\%) of the patients, respectively. Furthermore, the number of CTCs detected was specifically associated with bone metastasis, and the mean number of CTCs detected in patients with bone metastases was 5.7. ii) According to the histological features, patients with small-cell lung cancer (SCLC) displayed the highest CTC counts and a CTC detection rate of $77.8 \%$ (7 of 9 patients; median, 5; mean, 8.22) compared with patients with NSCLC or other types of metastatic diseases $(P>0.05$; Fig. 3A). iii) No significant association was observed between a higher CTC number and tumor burden or smoking status; however, a clear trend toward a correlation between an increased CTC detection rate and increased tumor diameter was observed ( $<3 \mathrm{~cm}, 48.7 \%$ vs. $\geq 3 \mathrm{~cm}, 71.1 \%$; $\mathrm{P}=0.078$ ).

CNV analysis and single-cell EGFR mutation detection. The use of CTCs as a real-time liquid biopsy has attracted increasing attention. Accurate and reliable techniques for the capture of purified CTCs from peripheral blood are therefore required to facilitate the clinical utility of CTC analyses. WGA was performed for DNA amplification and to identify the genomic features of CTCs. The DNA was subsequently subjected to $\mathrm{CNV}$ analyses. Multiple chromosomal variations were observed in the H2009 lung cancer cell line at the single-cell level, compared with those in normal white blood cells. Representative CNV profiles of five patients are illustrated in Fig. 4. Tumor-characteristic CNVs were detected in four patients using 1-10 CTCs. Based on the sensitivity of 1/1,000 copies for dPCR and 1/100 copies for the amplification refractory mutation system (ARMS) procedures, our study confirmed that both dPCR- and ARMS-based assays were suitable for analyzing mutations in single H1975 or HCC-827 cells, as displayed in Fig. 5. A total of three other lung cancer cell lines were also used to verify that dPCR- and ARMS-based approaches were effective for the detection of expected EGFR mutations (Table II).

Homogeneity analysis of CTC-EGFR mutations. Initially, EGFR mutations were analyzed in single CTCs following WAG; two single cells carried the same EGFR mutations as the primary tumor among 12 single cells derived from different patients using dPCR- and ARMS-based detection methods, indicating a $16.67 \%$ sensitivity. When samples containing more CTCs (10 CTCs from four patients) were analyzed, all the samples displayed clear EGFR mutation status, consistent with the tumor tissue, including two patients with the L858R mutation, one patient with the 19del mutation, and one patient with a non-activating mutation. The two samples with the L858R mutation were further confirmed by dPCR.

Inconsistent rare EGFR mutations are identifiable in CTCs. In addition to the four patients described above, EGFR mutations were also detected in the remaining four samples, 
H2009
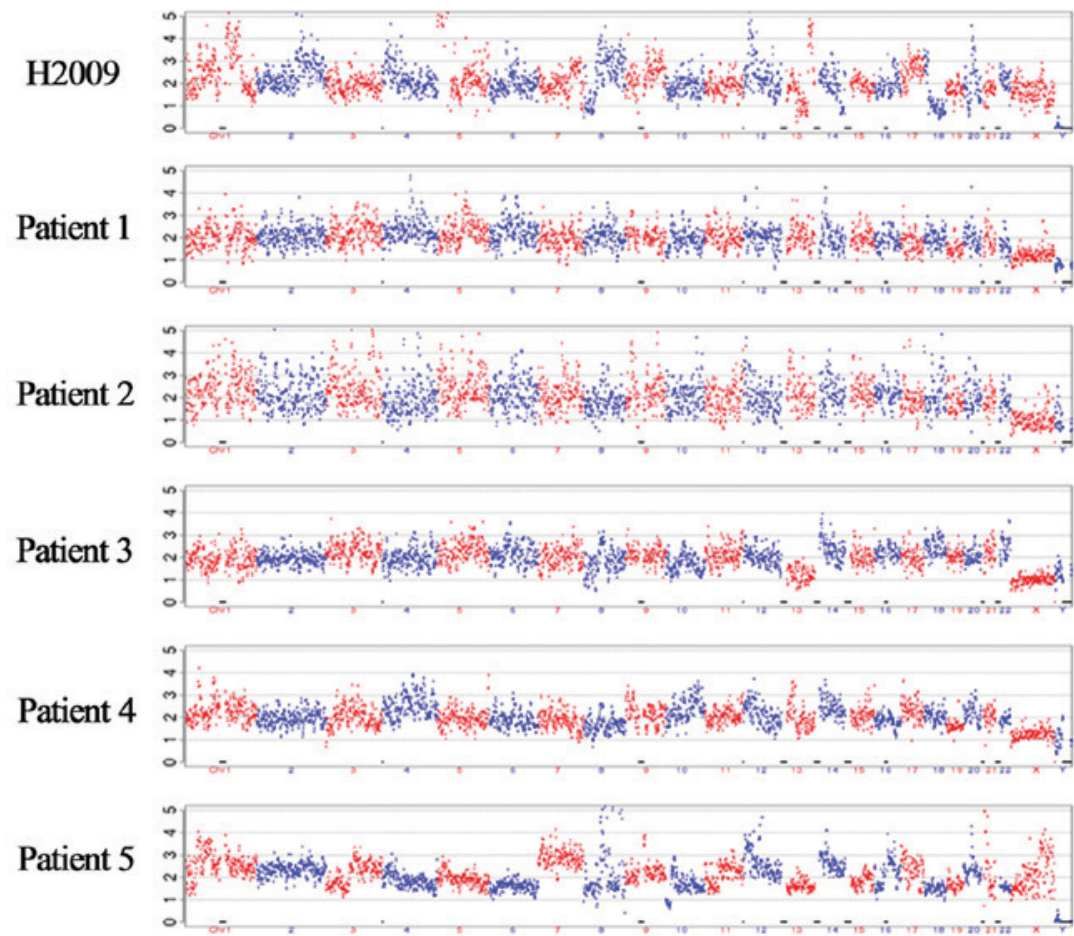

Figure 4. CNV analysis. Epithelial cell adhesion molecule+ and cytokeratin+ cells isolated from the peripheral blood of patients with lung cancer were used for whole-genome amplification followed by next-generation sequencing. Each panel represents 1-10 circulating tumor cells from different patients (patients 1-4) with confirmed CNVs, and CNVs in the $\mathrm{H} 2009$ lung cancer cell line were used as a positive reference. The CNV analysis was performed using the Cancer Gene Census database. CNV, copy number variation.
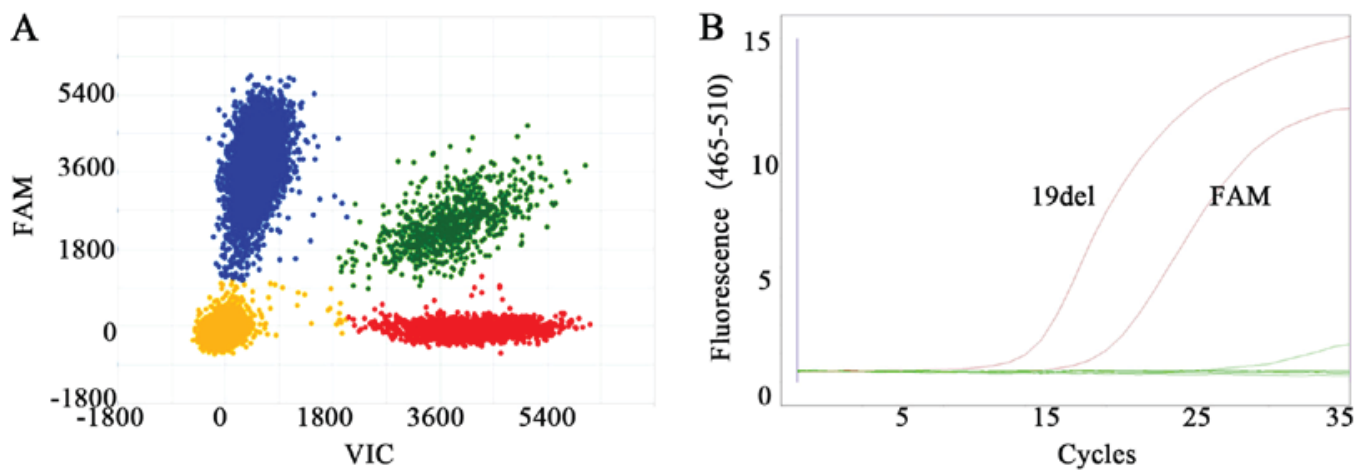

Figure 5. Detection of common EGFR mutations by dPCR and ARMS at the single-cell level. EGFR mutations L858R and 19del were detected in the amplified genomic DNA from single cancer cells (NCI-H1975 and HCC-827) by (A) 3D dPCR and (B) ARMS, respectively. dPCR, digital polymerase chain reaction; ARMS, amplification refractory mutation system; EGFR, epidermal growth factor receptor; FAM, 5-carboxyfluorescein; VIC, 2'-chloro-7' phenyl-1,4-dichloro-6-carboxy-fluorescein.

with CTC numbers ranging between three and nine cells. Among them, two samples yielded identical results to those of the tissue, while one sample contained four CTCs from the patient (patient 2) that were detected as wild type, which was a different result from the tissue pathological examination that revealed a 19del mutation. Another sample from patient 4 contained three CTCs in which a single new G791x mutation was detected that differed from the L858R mutation that was observed in this patient's tumor tissue (Table III).

\section{Discussion}

A number of promising techniques, including the use of microfluidic and microarray devices, may potentially enrich
CTCs with greater sensitivity. However, these techniques have not been approved for clinical use, and their utility in the discovery of new CTC markers is in the early stages of development. The CellSearch ${ }^{\circledR}$ system has undergone extensive development and is an accredited method for analyzing CTCs $(12,13)$. Current technical challenges include methods of increasing CTC purity and isolating single CTCs for molecular analysis. Researchers universally acknowledge that no single marker is able to reliably identify CTCs. Mainstream techniques continue to focus on utilizing common epithelial cell-specific markers, including EpCAM and CKs, which were introduced as a basic principle by the CellSearch ${ }^{\circledR}$ system. A key issue in conducting the present study was isolating high-quality CTCs. Therefore, the aim was to isolate CTCs 
Table II. Lung cancer cell lines with the expected EGFR mutations and the EGFR mutations detected by digital polymerase chain reaction or amplification refractory mutation system.

\begin{tabular}{llll}
\hline & \multicolumn{2}{c}{ EGFR mutation } & Histological \\
\cline { 2 - 3 } Cell lines & \multicolumn{1}{c}{ Expected } & \multicolumn{1}{c}{ Detected } & cell type \\
\hline NCI-H2009 & Wild-type & Wild-type & Adeno \\
HCC-827 & 19del & 19del & Adeno \\
NCI-H1975 & L858RT790M & L858RT790M & Adeno \\
\hline
\end{tabular}

EGFR, epidermal growth factor receptor; Adeno, adenocarcinoma.

Table III. Analysis of the consistency of EGFR mutations among various CTCs and tissue specimens.

\begin{tabular}{|c|c|c|c|c|c|}
\hline \multirow[b]{2}{*}{ Patient } & \multirow{2}{*}{$\begin{array}{c}\text { CTC } \\
\text { sample }\end{array}$} & \multirow{2}{*}{$\begin{array}{c}\text { CTC } \\
\text { number }\end{array}$} & \multicolumn{2}{|c|}{$\begin{array}{c}\text { EGFR } \\
\text { mutation (ARMS) }\end{array}$} & \multirow[b]{2}{*}{ Matched } \\
\hline & & & Tissue & CTC & \\
\hline \multirow[t]{2}{*}{1} & 1 & 10 & L858R & L858R & Yes \\
\hline & 2 & 9 & L858R & L858R & Yes \\
\hline \multirow[t]{2}{*}{2} & 1 & 10 & 19del & 19del & Yes \\
\hline & 2 & 4 & 19del & $\mathrm{N}$ & No \\
\hline \multirow[t]{2}{*}{3} & 1 & 10 & $\mathrm{~N}$ & $\mathrm{~N}$ & Yes \\
\hline & 2 & 3 & $\mathrm{~N}$ & $\mathrm{~N}$ & Yes \\
\hline \multirow[t]{2}{*}{4} & 1 & 10 & L858R & L858R & Yes \\
\hline & 2 & 3 & L858R & G719X & No \\
\hline
\end{tabular}

CTC, circulating tumor cell; EGFR, epidermal growth factor receptor; ARMS, amplification refractory mutation system.

from blood cells based on epithelial cell-specific markers. The system would need to be simple and automated for widespread clinical use; however, manual CTC retrieval under the microscope was an essential step to ensure the capture of single or pure CTCs. The CTC isolation technique was tested using $4.5 \mathrm{ml}$ of blood in spiking experiments and exhibited a recovery rate between $62.5-83.8 \%$, more efficient than that of previous reports on CTC detection using $7.5 \mathrm{ml}$ blood (8). This increase in capture efficiency may be attributed to double staining for EpCAM and CKs, as EpCAM is not expressed by all carcinomas, and as many as $30 \%$ express low levels of EpCAM. Moreover, the expression levels of CKs vary among different histological subtypes $(14,15)$. Simultaneous staining with multiple epithelial markers and confirmation of negative staining for leukocyte markers such as CD45, has become the standard for reliably identifying CTCs (16). Additionally, CTCs with low EpCAM expression have also been detected using a similar capture system (17). Among patients with lung cancer, the CTC count significantly increased in advanced tumor, such as SCLC and tumor progression, particularly with the development of distant metastasis. These findings are consistent with previous reports $(18,19)$.
The CellSearch ${ }^{\circledR}$ system has indicates promise for reliable and reproducible CTC enumeration in the clinic, and it has been approved for the evaluation of lung cancer. Patients without clinically detectable distant metastases will likely exhibit low CTC detection rates (8). Therefore, 91 patients with stage III and IV diseases were enrolled to evaluate the efficacy of a CTC detection protocol. In general, the results were similar to previously reported findings; the CTC count significantly increased with tumor progression, with a detection rate of $69.3 \%$ among patients with advanced stage IV NSCLC $(18,19)$, and CTCs were most abundant in patients with SCLC, as CTCs were detected in $77.8 \%$ of patients with this type of cancer (20). A clear trend towards an increased CTC detection rate with increased tumor size was also observed, although the association between higher CTC numbers and greater tumor size was not statistically significant. A detailed analysis of additional samples may be required to verify the association between CTC numbers and the actual tumor burden, rather than primary tumor size (volume). In contrast to a microfluidic device-confirmed CTC cluster, no CTC clusters were isolated from the 91 patients using our established protocol; this result was consistent with those from other reports using the CellSearch ${ }^{\circledR}$ system. It was concluded that in the present study, a simplified protocol using double staining resulted in a higher CTC capture rate and is suitable for clinical use, including single-cell detection. Moreover, previous studies have confirmed the prognostic significance of CTCs in patients with lung cancer, indicating that the CTC number is an independent prognostic factor for SCLC at baseline and following chemotherapy $(19,20)$.

Notably, ctDNA has been identified as a specific and sensitive biomarker for the detection of EGFR mutations (21-23). ctDNA is a part of the total circulating-free DNA, comprising small fragments of between 70 and 200 base pairs, and is released from dead cancer cells via apoptosis, necrosis and other mechanisms that remain to be clarified (24). The detection of ctDNA is also a characteristic of tumors with high cellular turnover. Cell-free ctDNA is frequently evaluated in non-invasive, early diagnostic screens to monitor responses and disease prognosis and to monitor resistance to treatment (25). In this regard, the biology of CTCs will hopefully be better understood with the availability of more comprehensive molecular characterization data, and information on their clinical implications (26).

In the clinic, decisions regarding therapy rely heavily on local tumor analyses. Biopsies of primary lesions are invasive procedures; therefore, an analysis of CTCs as representative surrogates for primary and metastatic lesions may be a potential alternative that may also enable the real-time assessment of resistance to therapy (27). Deep-sequencing genomic analyses of CTCs from primary tumors and metastases in patients with colorectal or prostate cancer have revealed that mutations in CTCs resemble those detected in primary tumors and metastases. These data have important implications for the use of CTCs as a liquid biopsy $(13,27,28)$. Using a microfluidic device, the expected activating EGFR mutations identified in the original tumor biopsy specimens have also been detected in CTCs from 11 of 12 patients with lung cancer (92\%) (4). More recently, a novel in vivo device was used to capture circulating lung cancer cells from one patient, and the same EGFR mutations identified in the primary tumors were clearly detectable in the collected CTCs (29). In the present 
study, detection of EGFR mutations at the single-cell level was initially attempted; however, this strategy was less effective, as only $\sim 16 \%$ of cells were positive for mutations in the analysis of 12 single cells derived from different patients with a known primary mutation. A subsequent study revealed that a sample with 10 CTCs exhibited an increased detection rate and proved effective in detecting the mutation identified in the primary tumor. Another study reported a similar result of a low percentage of EGFR mutations in CTCs analyzed using next-generation sequencing (30). Based on these results, as little as one CTC is appropriate for detecting EGFR mutations, and when the CTC number is decreased, sensitivity decreases. Generally, EGFR mutations were homogeneous. However, we observed one notable exception, in which a clear rare mutation was detected that differed from the primary mutation.

Although the present study is limited by the small sample size, particularly for the EGFR mutation analysis, the preliminary results support an expanded study using isolated CTCs to detect EGFR mutations and a potent heterogeneity analysis of somatic copy number alterations and mutations.

\section{Acknowledgements}

Not applicable.

\section{Funding}

This study was supported by grants of "Study on the Application of Clinical Characteristics of Capital" to HTZ (no Z151100004015104) and to SCZ (no. Z161100000516107) from Beijing Municipal Science and Technology Project.

\section{Availability of data and materials}

The datasets used and/or analyzed during the current study are available from the corresponding author on reasonable request.

\section{Authors' contributions}

Conceived and designed the experiments: SCZ, HTZ and QZ. Performed the experiments: QZ, JYN, ZHY and LY. Analyzed the data: QZ, JYN, ZDL and JHW. Contributed reagents/ materials/analysis tools: QZ, ZDL and JYN. Wrote the paper: QZ, SCZ and HTZ. Discussed the results and implications and commented on the manuscript at all stages: QZ, SCZ and HTZ.

\section{Ethical approval and consent to participate}

The study involved taking peripheral blood from healthy human, which was approved by Beijing Tuberculosis and Thoracic Tumor Research Institute Ethics Committee. All participants provided written informed consent prior to participation in the study.

\section{Patient consent for publication}

Patients consented to the publication of their data.

\section{Competing interests}

The authors declare that they have no competing interests.

\section{References}

1. Siegel RL, Miller KD and Jemal A: Cancer statistics, 2017. CA Cancer J Clin 67: 7-30, 2017.

2. Carmichael J, Wing-san Mak D and O Brien M: A review of recent advances in the treatment of elderly and poor performance NSCLC. Cancers (Basel) 10: pii: E236, 2018.

3. Nagano T, Tachihara M and Nishimura Y: Mechanism of resistance to epidermal growth factor receptor-tyrosine kinase inhibitors and a potential treatment strategy. Cells 7: pii: E212, 2018.

4. Cooper MR, Alrajhi AM and Durand CR: Role of immune checkpoint inhibitors in small cell lung cancer. Am J Ther 25: e349-e356, 2018.

5. Rosell R, Moran T, Queralt C, Porta R, Cardenal F, Camps C, Majem M, Lopez-Vivanco G, Isla D, Provencio M, et al: Screening for epidermal growth factor receptor mutations in lung cancer. N Engl J Med 361: 958-967, 2009.

6. Wu YL, Zhong WZ, Li LY, Zhang XT, Zhang L, Zhou CC, Liu W, Jiang B, Mu XL, Lin JY, et al: Epidermal growth factor receptor mutations and their correlation with gefitinib therapy in patients with non-small cell lung cancer: A meta-analysis based on updated individual patient data from six medical centers in mainland China. J Thorac Oncol 2: 430-439, 2007.

7. Maheswaran S, Sequist LV, Nagrath S, Ulkus L, Brannigan B, Collura CV, Inserra E, Diederichs S, Iafrate AJ, Bell DW, et al: Detection of mutations in EGFR in circulating lung-cancer cells. N Engl J Med 359: 366-377, 2008.

8. Allard WJ, Matera J, Miller MC, Repollet M, Connelly MC, Rao C, Tibbe AG, Uhr JW and Terstappen LW: Tumor cells circulate in the peripheral blood of all major carcinomas but not in healthy subjects or patients with nonmalignant diseases. Clin Cancer Res 10: 6897-6904, 2004.

9. Hou J, Greystoke A, Lancashire L, Cummings J, Ward T, Board R, Amir E, Hughes S, Krebs M, Hughes A, et al: Evaluation of circulating tumor cells and serological cell death biomarkers in small cell lung cancer patients undergoing chemotherapy. Am J Pathol 175: 808-816, 2009.

10. $\mathrm{Li} \mathrm{H}$ and Durbin R: Fast and accurate short read alignment with burrows-wheeler transform. Bioinformatics 25: 1754-1760, 2009.

11. Li H and Durbin R: Fast and accurate long-read alignment with burrows-wheeler transform. Bioinformatics 26: 589-595, 2010.

12. Parkinson DR, Dracopoli N, Petty BG, Compton C, Cristofanilli M, Deisseroth A, Hayes DF, Kapke G, Kumar P, Lee JSH, et al: Considerations in the development of circulating tumor cell technology for clinical use. J Transl Med 10: 138, 2012.

13. Alix-Panabières $C$ and Pantel K: Challenges in circulating tumour cell research. Nat Rev Cancer 14: 623-631, 2014.

14. Went PT, Lugli A, Meier S, Bundi M, Mirlacher M, Sauter G and Dirnhofer S: Frequent EpCam protein expression in human carcinomas. Hum Pathol 35: 122-128, 2004.

15. Broers JL, Ramaekers FC, Rot MK, Oostendorp T, Huysmans A, van Muijen GN, Wagenaar SS and Vooijs GP: Cytokeratins in different types of human lung cancer as monitored by chain-specific monoclonal antibodies. Cancer Res 48: 3221-3229, 1988.

16. O Flaherty JD, Gray S, Richard D, Fennell D, O Leary JJ, Blackhall FH and O Byrne KJ: Circulating tumour cells, their role in metastasis and their clinical utility in lung cancer. Lung Cancer 76: 19-25, 2012.

17. Punnoose EA, Atwal SK, Spoerke JM, Savage H, Pandita A, Yeh RF, Pirzkall A, Fine BM, Amler LC, Chen DS and Lackner MR: Molecular biomarker analyses using circulating tumor cells. PLoS One 5: e12517, 2010.

18. Krebs MG, Sloane R, Priest L, Lancashire L, Hou JM, Greystoke A, Ward TH, Ferraldeschi R, Hughes A, Clack G, et al: Evaluation and prognostic significance of circulating tumor cells in patients with non-small-cell lung cancer. J Clin Oncol 29: 1556-1563, 2011.

19. Tanaka F, Yoneda K, Kondo N, Hashimoto M, Takuwa T, Matsumoto S, Okumura Y, Rahman S, Tsubota N, Tsujimura T, et al: Circulating tumor cell as a diagnostic marker in primary lung cancer. Clin Cancer Res 15: 6980-6986, 2009.

20. Hou J, Krebs MG, Lancashire L, Sloane R, Backen A, Swain RK, Priest LJ, Greystoke A, Zhou C, Morris K, et al: Clinical significance and molecular characteristics of circulating tumor cells and circulating tumor microemboli in patients with small-cell lung cancer. J Clin Oncol 30: 525-532, 2012.

21. Thress KS, Brant R, Carr TH, Dearden S, Jenkins S, Brown H, Hammett T, Cantarini M and Barrett JC: EGFR mutation detection in ctDNA from NSCLC patient plasma: A cross-platform comparison of leading technologies to support the clinical development of AZD9291. Lung Cancer 90: 509-515, 2015. 
22. Bordi P, Del Re M, Danesi R and Tiseo M: Circulating DNA in diagnosis and monitoring EGFR gene mutations in advanced non-small cell lung cancer. Transl Lung Cancer Res 4: 584-597, 2015.

23. Qiu M, Wang J, Xu Y, Ding X, Li M, Jiang F, Xu L and Yin R: Circulating tumor DNA is effective for the detection of EGFR mutation in non-small cell lung cancer: A meta-analysis. Cancer Epidemiol Biomarkers Prev 24: 206-212, 2015

24. Jahr S, Hentze H, Englisch S, Hardt D, Fackelmayer FO, Hesch RD and Knippers R: DNA fragments in the blood plasma of cancer patients: Quantitations and evidence for their origin from apoptotic and necrotic cells. Cancer Res 61: 1659-1665, 2001.

25. Alix-Panabières $\mathrm{C}$ and Pantel K: Circulating tumor cells: Liquid biopsy of cancer. Clin Chem 59: 110-118, 2013

26. Siravegna G, Marsoni S, Siena S and Bardelli A: Integrating liquid biopsies into the management of cancer. Nat Rev Clin Oncol 14: 531-548, 2017.

27. Effenberger KE, Schroeder C, Eulenburg C, Reeh M, Tachezy M, Riethdorf S, Vashist YK, Izbicki JR, Pantel K and Bockhorn M: Disseminated tumor cells in pancreatic cancer-an independent prognosticator of disease progression and survival. Int J Cancer 131: E475-E483, 2012.
28. Nagrath S, Sequist LV, Maheswaran S, Bell DW,Irimia D, Ulkus L, Smith MR, Kwak EL, Digumarthy S, Muzikansky A, et al: Isolation of rare circulating tumour cells in cancer patients by microchip technology. Nature 450: 1235-1239, 2007.

29. Gorges TM, Penkalla N, Schalk T, Joosse SA, Riethdorf S, Tucholski J, Lücke K, Wikman H, Jackson S, Brychta N, et al: Enumeration and molecular characterization of tumor cells in lung cancer patients using a novel in vivo device for capturing circulating tumor cells. Clin Cancer Res 22: 2197-2206, 2016.

30. Marchetti A, Del Grammastro M, Felicioni L, Malatesta S, Filice G,Centi I, De Pas T, Santoro A, Chella A, Brandes AA, et al: Assessment of EGFR mutations in circulating tumor cell preparations from NSCLC patients by next generation sequencing: Toward a real-time liquid biopsy for treatment. PLoS One 9: e103883, 2014.

(i) $(-)$ This work is licensed under a Creative Commons Attribution-NonCommercial-NoDerivatives 4.0 International (CC BY-NC-ND 4.0) License. 